\title{
Reconocimiento de victimas del conflicto armado en Colombia: Sobre tecnologias de representación y configuraciones de Estado ${ }^{1}$
}

\author{
Fredy Mora-Gámez ${ }^{2}$ \\ Universidad de Leicester, Leicester, Reino Unido ${ }^{3}$ \\ Universidad Nacional de Colombia, Bogotá, Colombia ${ }^{4}$ \\ famorag@unal.edu.co \\ Recibido: 29 de julio de 2015 \\ Aprobado: 30 de octubre de 2015 \\ Disponible en línea: 9 de mayo de 2016
}

\footnotetext{
Artículo de investigación derivado de la tesis doctoral "Between victimhood and statehood: sociotechnical mediations for rights restitution of victims of the armed conflict in Colombia"

2 Magíster en estudios sociales de la ciencia de la Universidad Nacional de Colombia, 2012

3 Candidato doctoral de la Universidad de Leicester (Reino Unido)

4 Investigador del Grupo de Estudios Sociales de la Ciencia, la Tecnología y la Medicina de la Universidad Nacional
} 


\title{
Reconocimiento de victimas del conflicto armado en Colombia: Sobre tecnologias de representación y configuraciones de Estado
}

\section{Resumen}

Este trabajo aborda los procedimientos expertos y administrativos implicados en el Registro Único de Víctimas (RUV) a través del rastreo etnográfico de las prácticas alrededor del Formato Único de Declaración (FUD) que materializan el reconocimiento de víctimas del conflicto armado en Colombia. En este sentido, el RUV y el FUD se problematizan como tecnologias que reconfiguran al Estado colombiano y se describen algunas de sus trayectorias. Esta investigación discute la forma en que el reconocimiento de víctimas transforma las experiencias de quienes son reconocidos y quienes reconocen, explorando también una aproximación a la conformación del Estado precisamente desde las prácticas que ensamblan una noción particular de víctima que se intersecta con distintos proyectos estatales.

Palabras clave: reconocimiento de víctimas; ley de víctimas; etnografia del Estado; estudios sociales de la ciencia

\section{Acknowledging Victims of the Armed Conflict in Colombia: On the Representation Technologies and State Configurations}

\begin{abstract}
This work addresses the expert and administrative procedures implied in the Unique Victim Registry (RUV) by means of the ethnographic tracking of the practices surrounding the Unique Statement Form (FUD), which materializes acknowledgement of the victims of the armed conflict in Colombia. In this regard, the RUV and FUD are questioned as technologies which reconfigure the Colombian State and a part of their trajectories is described. This research discusses how acknowledging the victims transforms the experiences of those who are acknowledged and those who acknowledge. Also, it explores an approach to the structure of the State, particularly from the practices which configure a particular notion of victim met in the different state projects.
\end{abstract}

Keywords: acknowledgement of victims; victims law; State ethnography; social studies of science

\section{Reconhecimento de vitimas do conflito armado na Colômbia: Sobre tecnologias de representação e configurações de Estado}

\section{Resumo}

Este trabalho aborda os procedimentos especialzados e administrativos envolvidos no Registro Único de Vítimas (RUV) através do rastreio etnográfico de práticas ao redor do Formulário Único de Declaração (FUD) que materializam o reconhecimento de vítimas do conflito armado na Colômbia. Nesse sentido, o RUV e o FUD problematizam-se como tecnologias que reconfiguram o Estado colombiano e algumas das suas trajetórias são descritas. Esta pesquisa discute a forma em que o reconhecimento de vitimas transforma as experiências de quem são reconhecidos e quem reconhecem, explorando também uma aproximação à conformação do Estado precisamente desde as práticas que ensamblam uma noção particular de vítima que se intersecta com diferentes projetos estatais.

Palavras-chave: reconhecimento de vítimas; lei de vítimas; etnografia do Estado; estudos sociais da ciência 
A partir de los cambios administrativos derivados de la Ley 1448 de 2011, también conocida como Ley de Víctimas y Restitución de Tierras, el gobierno de Colombia ha desplegado un número importante de expertos de distintas disciplinas sociales con el fin de ejecutar procedimientos estandarizados de identificación de personas afectadas por el conflicto armado a través del Registro Único de Víctimas (RUV). Dicho grupo de expertos está a cargo de implementar medidas de acogida y asistencia dirigidas a personas que desean ser incluidas en el RUV, para lo cual se han desarrollado herramientas técnicas como el Formato Único de Declaración (FUD). El presente trabajo rastrea etnográficamente las trayectorias del FUD y las experiencias de declarantes y funcionarios en espacios como las Unidades de Atención y Orientación (UAO), los Centros Dignificar/Regionales y las oficinas gubernamentales a cargo del reconocimiento de víctimas del conflicto armado en Colombia.

Particularmente sobre las situaciones de justicia y derechos como las que pretende documentar el presente estudio, Honneth (2003) sugiere que el reconocimiento surge de la "[...]comprensión del estatus de igualdad de los otros como personas autónomas [...]" (p. 151) y resulta en el establecimiento de identidades que gozan de legitimación, derechos y bienes. A partir de una crítica al concepto de comprensión sugerido por Honneth, Rancière (1998) señala que el reconocimiento de sujetos acreedores de derechos únicamente es posible cuando se cuestiona el supuesto de igualdad que sustenta el orden jerárquico de la sociedad. En este sentido, la noción de reconocimiento sugerida por Honneth al parecer, legitima precisamente el orden estatal del cual el individuo reconocido se hace parte; podríamos pensar entonces que los actos de reconocimiento oficial se configuran a partir de relaciones en donde la subjetividad de quien es reconocido es transformada por un ente reconocedor como el Estado.

En sintonía con Ranciere pero para el caso particular de personas en situación de inmigración, Papadopoulos, Stephenson y Tsianos. (2008) han sugerido que el reconocimiento es un acto de representación, en el cual las personas son incorporadas dentro de sistemas formales que garantizan el control sobre su ubicación, movilidad y formas de empleo a cambio del goce de ciertos derechos. Por tanto el equilibrio entre 
representación y derechos (conocido también como el axioma $\mathrm{R}-\mathrm{R}$ ) es una condición para la soberanía de los Estados. (p. 6) Esta última aproximación al reconocimiento como representación resulta de particular relevancia para el abordaje del presente artículo en tanto que incorpora la participación de sistemas de registro y gestión como mediadores del reconocimiento de derechos por parte del Estado.

En este estudio indago por el sistema técnico que materializa la representación de personas que buscan su inscripción en el RUV a través de las rutas señaladas por el Estado colombiano. Dicho sistema incorpora actores, objetos y formas de experticia, por lo cual se constituye en un ensamblado socio-técnico (Latour, 2005) en tanto posibilita relaciones, moviliza formas de experticia y materializa una noción estatal del reconocimiento de víctimas. Dentro del abordaje de tales ensamblados, las etnometodologías constituyen una importante tradición para dar cuenta de la configuración entre saberes expertos y otras formas de orden social centrándose en las trayectorias de actores y objetos (Hess, 2001).

Algunos reportes etnográficos que problematizan el rol de la experticia en la atribución de derechos por parte de entidades gubernamentales son presentados por Petryna (2002) sobre las compensaciones de habitantes de las zonas cercanas a Chernobyl luego del desastre y Biehl, Good y Kleinman (2007) documentan las experiencias de personas de escasos recursos en Brasil al reclamar atención del sistema de salud para sus diagnósticos psiquiátricos. De forma más pertinente, para casos de violencia con motivos políticos, algunos abordajes etnográficos centrados en actores y sus interacciones con instituciones han sido presentados por Harrell-Bond (1986) quien destaca las prácticas administrativas que resultan en la imposición de ayudas humanitarias para refugiados en distintas zonas de África, De forma semejante Boyden y De Berry (2004) documentan las experiencias de niños, niñas y adolescentes durante situaciones de guerra.

Considerando que la representación socio-técnica de personas en el RUV comprende un proyecto estatal en curso, su abordaje etnográfico como ensamblado socio técnico se intersecta con los intereses de distintas etnografias del Estado al intentar aproximarse a las prácticas 
de conformación de racionalidades y ordenes sociales que constituyen el Estado mismo. Los abordajes del Estado como problema etnográfico se enriquecen de importantes trabajos en Latinoamérica sobre los efectos de la ley y del Estado en Colombia (Sanford 2004 cit. por Schavelzo, 2010), la relación entre burocracias y violencia (Tiscornia 2004) y la gestión de la política en programas de vivienda en Argentina (Schavelzon 2007), entre otros. En estas aproximaciones se destaca de forma frecuente el valor de la descripción de las prácticas administrativas como acciones de Estado y como muestras de la distribución del poder estatal o del ejercicio del poder por parte de actores en las instituciones.

Como lo señala Schavelzon (2010) sobre la antropología, aunque en un sentido amplio de las aproximaciones etnográficas a las prácticas estatales, el Estado aparece como un objeto en el que es posible observar fenómenos culturales donde

[...] lo externo al Estado, lo ilegal, lo marginal y lo tradicional aparecen [ahora] no necesariamente en espacios geográficos lejanos o no alcanzados por el mismo, sino como elementos presentes en el centro de nuestro sistema político moderno y estatal. (p. 20)

Desde una aproximación etnográfica al Estado y a los ensamblados que permiten su conformación, el siguiente trabajo problematiza las prácticas socio técnicas que median la representación de víctimas sobre la cual se construyen proyectos de orden social que reconfiguran el Estado colombiano. A continuación intentaré mostrar cómo la dimensión política del reconocimiento de víctimas se constituye en una dinámica socio técnica en donde se legitiman y promueven distintas formas de Estado a través de dispositivos como el FUD en espacios como los CDR y las oficinas de valoración.

\section{El FUD en los CDR}

Mis visitas a los Centros Dignificar/Regionales empezaron en 2013, dos años después de la promulgación de la Ley de Víctimas. Estas 
oficinas se encuentran en distintas ciudades del país y largas filas tienden a congregarse con frecuencia en frente de sus instalaciones desde tempranas horas del día. Uno de los primeros encuentros lo sostengo con Mario, un campesino de Norte de Santander quien acaba de llegar a la ciudad; indago entonces por el propósito de las filas en los centros de atención.

Mario: Ah, eso es para responder unas preguntas.

Fredy: ¿Recuerda alguna de las preguntas?

M: Sí bueno, mi edad, el nombre de mi esposa, los números de cédula, el certificado de nacimiento de mi hija, si sabía quién me había amenazado y por qué, eso [...] si tenía familia en Bogotá, la fecha en que salí, la fecha de cuando llegué a Bogotá, la fecha de la amenaza [...] ella como que me preguntó mucho por fechas y lugares [...] supongo que tienen que estar seguros, pero no sé [...]

F: ¿Seguros?

M: Creo que estaba como probándome, como chequeando la información varias veces pero bueno, no sé, supongo que tienen que probar a todo el mundo, ¿̇o no?

Además de señalar la repetición de preguntas sobre fechas y lugares, y de manifestar cierta comprensión ante la necesidad de comprobación por parte de los funcionarios públicos, en esta conversación Mario emplea la palabra ella para referirse a profesionales del ministerio público como abogados, trabajadores sociales y psicólogos, entre otros expertos a cargo de la recepción de declarantes que desean ser registrados como víctimas. Al final de su encuentro con una funcionaria, Mario recibió algunos bonos de mercado y un desprendible que intercambiaría por asistencia médica. El desprendible que Mario me enseña perteneció inicialmente al FUD que la funcionaria llenó por Mario al tiempo que formulaba un grupo extenso de preguntas y solicitaba documentos que probaran las afirmaciones de Mario. 
Según distintos funcionarios de la alcaldía de Bogotá y la Unidad para la Atención y Reparación Integral a las Víctimas (UARIV) la versión más reciente del FUD fue diseñada con anterioridad al inicio de la implementación de la Ley de Víctimas por administradores, psicólogos y trabajadores sociales convocados por la propia UARIV. Tal formato es el mecanismo oficial a través del cual las personas que desean ser reconocidas como víctimas del conflicto armado pueden ser incluidas en el RUV, lo cual precede el acceso a medidas de reparación que ha dispuesto el Estado colombiano a partir de la Ley de Víctimas (UARIV, 2013). Además de recoger información de tipo demográfico, a través de cuatro páginas y trece anexos, el FUD instruye a los funcionarios públicos para recolectar todas las pruebas documentales que sean posibles de las narraciones de los declarantes (Cruz Roja Colombiana, 2012). El FUD también solicita que se realice una evaluación general de las condiciones médicas y psicológicas cuyas anotaciones deben consignarse en los anexos respectivos.

De acuerdo con los lineamientos del FUD, debe haber una entrevista inicial antes de la narración de eventos de tal forma que el evaluador conozca con anterioridad la información que debe consignarse y las pruebas que se deben suministrar. Asimismo se solicitan pruebas de reportes a las autoridades hechas por los declarantes o sus familias, certificados de asistencia médica o psicológica, títulos de propiedad, pruebas de dirección, entre otras. La narración de eventos tiene lugar solo después de un procedimiento de codificación, insistencia en fechas, lugares, pruebas y circunstancias. Dentro de la misma lógica, el FUD insiste en orientar la narración de eventos para ofrecer una "declaración completa, consciente y coherente de los hechos", utilizando las preguntas sugeridas para cada anexo (Cruz Roja Colombiana, 2012). Los lineamientos también señalan la necesidad de "[...] registrar todos los detalles afectivos, emocionales, cognitivos y conductuales que el declarante experimenta en relación con los hechos, así como las expresiones relacionadas con su estado de ánimo durante la narración [...]". (DPS, 2012, p. 59).

En relación con los criterios para determinar la veracidad de una declaración, distintos funcionarios trabajando en Centros Dignificar/ Regionales (CDR) señalan la importancia de la rigurosidad durante 
el diligenciamiento del FUD puesto que dicha información permitiria una mejor evaluación de la solicitud de inclusión en el RUV. Esta evaluación se traduce en tiempos de espera de 90 días para Mario quien, luego de haber abandonado su finca por amenazas de grupos armados, manifiesta su preocupación por tener que esperar más de tres meses por una respuesta. Durante este tiempo pretende sobrevivir con cinco bonos de mercado que recibió y la garantía de una estadía de quince días en un albergue de la Cruz Roja. Mario comenta: "me imagino que se toman todo ese tiempo para hacer toda la investigación del caso, he sabido que llaman, o van a los lugares para averiguar que ‘jue[sic] lo que pasó con uno" y explica que tiene la intención de conseguir un trabajo que le permita sostenerse; sin embargo no debe buscar empleos permanentes que ofrezcan beneficios sociales antes de recibir una respuesta oficial, puesto que su aplicación al RUV sería retirada o rechazada al figurar en las bases de datos de beneficios sociales.

Las anteriores experiencias constituyen en buena medida la cotidianidad de declarantes y funcionarios que interactúan en espacios regulados por la Ley de Víctimas. El papel central de los formatos y las visitas a los CDR me recuerdan el trabajo de Mathew Hull (2012) sobre la producción de formas de orden gubernamental a través de la circulación de distintos artefactos textuales y burocráticos en Pakistán. Con una narrativa fiel a los detalles y circulaciones de formatos entre burócratas, políticos, campesinos y constructores que resultan en la construcción arquitectónica de la ciudad de Islamabad a finales de la década de 1990, el trabajo de Hull analiza la configuración de formas de gobernabilidad destacando la participación de los formatos en la construcción de un nuevo régimen. Aunque el estudio realizado en la localidad pakistaní se enfoca en la construcción arquitectónica de la ciudad de Islamabad, de forma análoga me inspira a preguntarme por la construcción experta de la noción de víctima de acuerdo con las regulaciones y por los proyectos estatales cuyas piedras angulares se cimientan en las materialidades del FUD.

Durante otra visita a un CDR puedo presenciar el encuentro entre una declarante a quien podré entrevistar posteriormente y un funcionario público a cargo del diligenciamiento del FUD. Desde la sala de espera en 
la que me encuentro acompañando a otra declarante y gracias al arreglo espacial del CDR que estoy visitando, puedo apreciar como la secuencia de preguntas por parte del funcionario sigue al pie de la letra el instructivo del FUD y las mismas preguntas son formuladas de distintas maneras una y otra vez. Mientras tanto las anotaciones del funcionario sobre el FUD transforman afirmaciones en códigos, frases breves y en palabras de la declarante, 'casillas marcadas'. De esta forma, la historia abrumadora relatada por la declarante es categorizada por el funcionario dentro de la taxonomía de hechos victimizantes ${ }^{5}$ que contiene el FUD. Como lo indica el libreto que he conocido con anterioridad, se deben formular preguntas específicas para cada código, así que luego de determinar que la declarante había recibido tratamiento psicológico, el funcionario debe marcar las casillas 'problemas en el discurso, la memoria y el razonamiento' además de 'trauma psicológico'.

Dentro del mismo encuentro el funcionario insiste en la necesidad de los documentos notariados, escrituras y evidencias de asistencia médica o psicológica para aumentar las posibilidades de inclusión en el registro, pero la declarante informa que no tiene muchos de esos documentos y que prefiere evitar el contacto con las personas de su ciudad. El encuentro termina luego de cuarenta minutos, cuando el funcionario pregunta si la declarante desea realizar algún cambio a su declaración, ante lo cual ella manifiesta que todo se encuentra en regla. Luego de que ambos firman e imprimen su huella, ella recibe un desprendible con un número único consecutivo que debe guardar para posteriores consultas. Además del desprendible se entregan bonos para mercado y la dirección de un albergue donde la declarante podrá permanecer durante quince días.

En lugares como los CDR los relatos de personas que deciden invocar la ayuda estatal se transforman en códigos, declaraciones y anotaciones dentro de un dispositivo que circulará en distintos espacios administrativos. Como se indicó anteriormente, estos arreglos

5 Códigos de hechos victimizantes: 1. Acto terrorista / atentados / combates / enfrentamientos / hostigamientos; 2. Amenaza; 3. Delitos contra la libertad y la integridad sexual en desarrollo del conflicto armado; 4. Desaparición forzada; 5. Desplazamiento forzado; 6. Homicidio / masacre; 7. Minas antipersonales, munición sin explotar y artefacto explosivo improvisado; 8. Secuestro; 9. Tortura; 10. Vinculación de niños, niñas y adolescentes en actividades relacionadas con grupos armados; 11. Abandono o despojo forzado de tierras; 12. Otro; 13. No es víctima (tomados del instructivo para el diligenciamiento del FUD, elaborado por la Unidad para la Atención y Reparación Integral de Victimas). 
que incorporan el conocimiento del evaluador, las traducciones que demanda el FUD y la participación de los declarantes conforman un ensamblado socio técnico (Latour, 2005) en donde el funcionario se configura como experto, el declarante como posible víctima, y el FUD como un mediador técnico (ver Latour, 1994). Dentro de estas mediaciones el FUD se posiciona como punto de paso obligatorio (Callon, 1986) parcialmente estable, incuestionado, diseñado inicialmente para gestionar el RUV y usado para determinar la veracidad de los relatos a partir de la consistencia de fechas y lugares.

Como lo señalan Callon y Latour (1981, p. 279) durante las dinámicas relacionales de traducción, los actores en ocasiones asumen la autoridad para 'hablar en nombre de otros'. Así, los expertos a través del diligenciamiento del FUD y las traducciones que demanda, se posicionan como voceros de los declarantes y de la misma manera, a partir de sus características agenciales-relacionales, el FUD será el objeto que represente a declarantes y funcionarios en otras trayectorias. En el mismo sentido Callon (1986, p. 216) señala que "hablar por otros consiste inicialmente en silenciar aquellos en cuyo nombre hablamos". De esta manera, podría pensarse que el ensamblado del FUD en los centros de asistencia silencia distintos aspectos como la experiencia dolorosa de quienes declaran, la incertidumbre de personas como Mario al intentar buscar un trabajo, entre otras voces, actores y actuantes. En este punto me pregunto por las traducciones y silenciamientos que tienen lugar en otras trayectorias del FUD dentro de las instancias estatales. Las movilizaciones de dicho dispositivo sugieren que el Estado se configura desde las propias prácticas administrativas. De la misma forma, las relaciones que se construyen en las prácticas administrativas alrededor de tecnologías como el FUD destacan el carácter emergente, interactivo e inestable del Estado así como la movilización de distintas formas de orden social que se requiere para tal conformación de Estado.

Con el fin de continuar el rastreo de las movilizaciones del FUD y su posterior consolidación en el RUV, a continuación presento algunas de las trayectorias documentadas en las oficinas gubernamentales donde los formatos son tramitados y valorados para emitir las comunicaciones oficiales sobre la inclusión en el registro. 


\section{La valoración del FUD}

En el área de recepción de los formatos y en compañía de dos funcionarios, puedo contemplar las cajas llenas de formatos que se acaban de recibir, en las cuales diversas firmas, fechas y registros de identificación se encuentran adheridos a la parte superior. Se alcanzan a distinguir formatos de Tolima, Quindio, Sucre, Norte de Santander, Huila y Magdalena. Resulta abrumador pensar en las historias detrás de esas cajas, en los años de experiencias de violencia, perpetraciones, abusos y tragedias representados en tales objetos que al mismo tiempo, me ponen en contacto con las personas que he acompañado en las filas. En este momento intento visualizar las secuencias de traducción que tuvieron lugar para que dichas historias ahora estén contenidas en un solo lugar para su evaluación experta.

Tales cajas y bases de datos quizás constituyen una forma de Panopticon (Foucault, 1983) burocrático en tanto el Estado colombiano maximiza su efectividad individualizando a los declarantes en formatos que a su vez los colocan en un estado de constante visibilidad. De forma semejante, la metáfora de James Scott (1998) que presenta al Estado como un organizador de sus habitantes a través del diseño de espacios y proyectos de desarrollo, también me permite comprender la magnitud del FUD como dispositivo de representación (ver Papadopoulos y Tsianos, 2013). Dentro de estos espacios las cajas, los formatos y las digitalizaciones de los mismos se constituyen como partes de la materialidad del propio Estado y de su proyecto de gestión de víctimas promulgado en la Ley 1448 de 2011 que promueve la gestión de las poblaciones que dicha regulación considera como afectadas por el conflicto armado.

Luego de ser recibidos y almacenados en la red interna, los FFUUDD son enviados al equipo de aproximadamente 130 profesionales psicosociales para su respectiva evaluación. La mayoría de aquellos profesionales tienen títulos en psicología, antropología, sociologia, trabajo social, ciencias politicas y algunos cuentan con formación de postgrado en derechos humanos. Las sesiones de valoración implementadas por los profesionales psicosociales ocurren con un libreto relativamente uniforme. Luego de recibir la asignación 
diaria de catorce FUD aproximadamente, los profesionales psicosociales consultan el número de cédula en distintas bases de datos para verificar si el declarante ya ha solicitado su inclusión en el RUV. De esta forma, bases de datos como la de la procuraduría para poblaciones desplazadas (SIPOD) son rápidamente revisadas para comprobar que los declarantes aún no han recibido compensaciones. Lo mismo ocurre con personas que acaban de declarar con su cédula pero habian sido incluidos como menores de edad en grupos familiares de declarantes bajo anteriores regulaciones. Al parecer, algo diferente ocurre con familiares de ex militantes de grupos al margen de la ley detectados en la base de datos de la Agencia Colombiana para la Reintegración (ACR). Como parte de las visitas, pude seguir un FUD que retrata tal situación y lo evalúa una profesional psicosocial, lo que me permite acompañarla con autorización de las directivas. Dichas descripciones son presentadas a continuación para dar cuenta de las fases de valoración del FUD que tienen lugar luego de los chequeos preliminares.

La primera fase se denomina Motivación, en ella se revisan los apartados literales de la declaración:

Considerando que XXXX en su declaración afirma: ‘[...] El no regresó a casa ese día y me preocupé mucho porque él nunca se queda por fuera. Al día siguiente a las 7 a.m. lo vi tirado en el suelo cerca a la casa [...]' Luego el declarante afirmó 'Los XXXX lo mataron porque él fue reclutado como menor pero luego se les escapó' $[\ldots]^{6}$ (nombres omitidos por la funcionaria a cargo)

La casilla del FUD conteniendo la categoría Homicidio es remarcada por la profesional psicosocial y funciona como un filtro selectivo para revisar la extensa narración de eventos. Luego la funcionaria ingresa el número de cédula del esposo de la declarante en la base de datos de la ACR y encuentra un registro. Así, el profesional psicosocial

6 Los extractos que se presentan sobre la valoración del FUD son tomados de un formato en evaluación. Todas las fechas, nombres y lugares fueron omitidos por las personas a cargo del cuidado y gestión de los formatos, siguiendo los lineamientos éticos de aprobación de este trabajo en la Universidad de Leicester y las pertinentes recomendaciones del personal de la UARIV para proteger la identidad de los declarantes así como el carácter confidencial de su información. 
procede a la siguiente fase denominada Jurídica, en la cual las resoluciones, sentencias, leyes y estamentos son citados para apoyar la decisión. Para el caso en cuestión el funcionario eligió el parágrafo 2 del artículo 3 de la Ley 1448.

[...] Para los efectos de la presente ley, el o la cónyuge, compañero o compañera permanente, o los parientes de los miembros de grupos armados organizados al margen de la ley serán considerados como víctimas directas por el daño sufrido en sus derechos en los términos del presente artículo [del asesinato de un familiar] pero no como víctimas indirectas por el daño sufrido por los miembros de dichos grupos $[\ldots]$ (subrayado del autor)

La funcionaria a cargo de la evaluación explica que en el caso en cuestión se reconoce que ocurrió un delito y los hechos que lo demuestran, pero no hay afectaciones que se consideren indemnizables en tanto el cónyuge asesinado no se había desmovilizado siendo menor de edad y tampoco hay evidencia suficiente de que el perpetrador sea un grupo armado.

Luego de esta explicación la funcionaria continúa con la fase de Contexto que consiste en referenciar los documentos oficiales y los informes sobre derechos humanos elaborados por fuentes gubernamentales como el programa presidencial para los derechos humanos, los diagnósticos de departamentos de 2000 a 2007 hechos por la Presidencia de la República, los informes de la Fiscalía General de la Nación, del Instituto Colombiano de Bienestar Familiar; los reportes de autoridades locales, la policía, las fuerzas militares y algunos medios de comunicación local. Para el caso en cuestión los reportes hechos por autoridades locales sobre crimenes de Estado fueron empleados para señalar que la narración era verdadera, sin embargo la veracidad de la declaración no garantizó el registro de la declarante en la fase técnica puesto que su caso representa una situación que escapa a la definición de víctima contemplada en la Ley de Víctimas. De esta forma, la notificación final dice: 
[...] Que al consultar las bases de datos de la Agencia Colombiana para la Reintegración -ACR- se encontró registro de XXX con el código XXXX [...] de los argumentos anteriores, se establece de manera objetiva y atendiendo rigurosamente el relato del declarante que el homicidio al que fue expuesto su cónyuge no se ocasionó por las circunstancias previstas en el artículo $3^{\circ}$ de la Ley 1448 de 2011, sino que fue ocasionado por circunstancias de tipo social y personal [...], este Despacho resuelve [...] NO INCLUIR en el Registro Único de Víctimas a XXX, identificada con cédula de ciudadanía XXX ni a los miembros de su hogar y NO RECONOCER el hecho victimizante de Homicidio, por las razones señaladas en la parte motiva de la presente resolución". (Notificación de no inclusión: fechas, nombres, números y lugares fueron omitidos por la funcionaria a cargo, subrayado del autor)

Luego de que distintos apartados de la resolución son copiados, pegados, redistribuidos y editados, el documento resultante es firmado digitalmente por el evaluador y su supervisor técnico para luego ser almacenado en los servidores de donde será descargado, impreso y enviado al centro de procedencia. De esta forma, los relatos que habian sido transformados en códigos y narraciones elaboradas dentro del FUD, ahora se configuran como casos exitosos de inclusión figurando en la cifra oficial del RUV movilizada por el gobierno, o como aplicaciones gestionadas que se condensan en indicadores de gestión. Dentro de las mismas secuencias de traducción, el reconocimiento estatal con base en criterios técnicos es otorgado como una resolución que informa a los declarantes sobre el resultado de su solicitud y les permite el inicio de la ruta de reparación. En el momento de las visitas que han permitido la presente investigación, los casi 130 profesionales psicosociales valoran y deciden sobre aproximadamente 26.000 solicitudes mensuales e incluyen en el RUV alrededor del $82 \%$ de las solicitudes (RNI, 2015). Si sumamos la cifra oficial existente en 2010 cercana a los 3.500 .000 , la decisión administrativa de incluir a las poblaciones desplazadas dentro del RUV y las solicitudes valoradas desde 2011 en la UARIV, para mayo de 2015 la cifra oficial de víctimas llega a 7.337.667 (UARIV, 2015). 


\section{Intringulis dentro del complejo FUD-RUV}

Encuentro de interés el uso del FUD como herramienta que intenta determinar la veracidad de las narraciones presentadas por los declarantes a través de la repetición de preguntas sobre fechas, eventos y lugares. Al parecer, la vigilancia de los recursos públicos como narrativa predominante en los funcionarios se constituye como un fundamento importante de tales prácticas de verificación, pero a la vez, dichas indagaciones revisan las afirmaciones naturalizadas por los declarantes. Sobre este punto Ashmore y Restrepo-Forero (2013) sugieren que los documentos en su paso por sistemas burocráticos como la notaria, son transformados en objetos de credibilidad, sin embargo señalan también que la notaria, en tanto espacio que coproduce un tipo de sociedad y formas específicas de lo social, produce efectos de confiabilidad y autenticidad, y que al interesarse en producir más confianza en cada procedimiento, la notaría "contribuye al vaciamiento de la confianza en la sociedad que la rodea” (Ashmore y Restrepo-Forero, 2013, p 425).

Para el caso particular del registro en el RUV, las narrativas de los declarantes codificadas en el FUD firmadas y selladas tienen mayor credibilidad ante la sociedad colombiana y los sistemas de evaluación, pero esto implica que tales ejercicios de contrastación y verificación como intentos de producir más veracidad de los relatos simultáneamente despojan de credibilidad a los relatos de aquellas personas que no son incluidos en el RUV. En sintonía con las profundas reflexiones sobre el reconocimiento político sugeridas por Ranciere (1998), el universo de personas que han sido afectadas por relaciones de violencia dentro del conflicto armado excede la noción de víctima que promueve y reconoce el Estado y por lo tanto sobrepasa los límites trazados por el ensamblado del RUV. En últimas, el FUD logra traducir las historias de algunos declarantes en relatos exitosos, verificables y legítimos al interior del sistema estatal, los cuales resultan en el reconocimiento como víctima, pero hay otras historias ausentes de pruebas documentales o que difieren de la noción estatal de víctima que se escapan al diseño del FUD y el RUV. 
Otro aspecto que resalto como crucial comprende la tensión en el tiempo de espera de tres meses para obtener una respuesta. Durante dicho periodo involucrarse en empleos con seguridad social supone el rechazo de sus declaraciones (Mora-Gámez, 2013). Como se puede determinar en las trayectorias rastreadas, los periodos de interacción entre los funcionarios a cargo de la recepción, digitalización, digitación y evaluación del FUD son reducidos, por lo cual durante el tiempo restante el FUD es trasladado y reposa en cajas o discos duros en espera de su evaluación. Aunque los funcionarios a cargo reconocen que el número de aplicaciones excede el sistema de gestión, quizás por los plazos para declarar en la Ley de Víctimas que entraban en caducidad en periodos cercanos a las visitas descritas en este texto, es cuestionable que un sistema de intervención supuestamente diseñado para mejorar o acompañar las dificiles situaciones de personas que han sido afectadas por relaciones de violencia, en efecto restrinja o condicione sus posibilidades de reorganización haciendo que el reconocimiento como víctima sea condicional a su situación laboral. Esta sobreestimación de la situación laboral y la empleabilidad como indicadores de restitución de derechos es predominante dentro del sistema estatal de reconocimiento y algunas de las estrategias posteriores de reparación. A pesar de lo anterior, el tiempo de espera tiene un efecto performativo en tanto los declarantes interpretan ese periodo como señal de investigación exhaustiva, evaluación objetiva y procedimientos cuidadosos que el Estado lleva a cabo para decidir sobre sus aplicaciones.

Anteriormente me referí al posicionamiento de funcionarios a cargo del diligenciamiento ante los declarantes como 'voceros' cuyas traducciones "inicialmente silencian a aquellos en cuyo nombre se habla" (Callon, 1986, p. 247). Así, podría pensarse que el ensamblado del FUD en los centros de asistencia silencia distintos aspectos como las experiencias dolorosas y la incertidumbre de diversos declarantes. En los espacios de valoración del FUD, un aspecto controversial corresponde a la consistencia entre las versiones suministradas por los declarantes con los reportes y bases de datos gubernamentales, de tal forma que las anotaciones hechas por los funcionarios que toman las declaraciones no determinan la inclusión en el RUV ni direccionan de forma importante la decisión del evaluador. Esto último contrasta las creencias de 
diversos funcionarios a cargo del diligenciamiento del FUD sobre la importancia de registrar los aspectos médicos y psicológicos. Me pregunto entonces por la utilidad de las anotaciones y observaciones elaboradas por los expertos en contacto directo con las personas. Si la decisión sobre el registro es un asunto de consistencia con las fuentes gubernamentales, entonces las bases de datos y una parte de la información del FUD son más que suficientes.

Intento pensar en un sistema alternativo en el que la recolección de información fuera efectuada por personas sin experticias acreditadas pero con entrenamiento en recolección de información, diligenciamiento de formatos y habilidades sociales de escucha y comprensión empática. En este experimento, los aspectos cruciales de la decisión se mantienen en fechas, lugares y eventos y su consistencia con las fuentes oficiales. Si como los evaluadores del FUD, silenciamos las partes que contienen los comentarios y anotaciones de los diligenciadores, un sistema conformado por funcionarios sin el tipo de experticia requerido actualmente podría cumplir la tarea de forma efectiva. Igualmente sucedería si decidiéramos sustituir a los expertos que evalúan el FUD por personas con entrenamiento en manejo de bases de datos, edición de documentos y una atención excepcional al detalle. Sin embargo, tal sistema hipotético probablemente fracasaría cuando las decisiones administrativas fueran objetadas públicamente o cuando los declarantes, la opinión pública u otros sectores cuestionaran la credibilidad del Estado, carente de expertos acreditados.

$\mathrm{El}$ anterior escenario nos sugiere otro efecto performativo de las experticias en el ensamblado del FUD, en tanto certifican y legitiman tal recolección de información promoviéndola como rigurosa y objetiva. Aunque las firmas de los funcionarios en distintas etapas del proceso también mantienen cierto grado de responsabilidad sobre los procedimientos administrativos, considero destacable el efecto legitimador de la experticia en el ensamblado de la noción de víctimas del conflicto armado movilizada por el Estado, naturalizando a su vez la supuesta racionalidad y justicia que subyace a dicha noción. A partir de lo anterior y emulando el proyecto cosmopolítico de Isabelle Stengers que propone superar las dicotomías entre hechos-valores, 
cuestiones objetivas-subjetivas y ciencia-politica (Stengers, 2002, pp. 23-24), podríamos repensar el FUD precisamente como un escenario científico político que configura una noción de víctima a través de sus procedimientos técnicos. Como se señaló anteriormente, el principal criterio de inclusión y veracidad se concentra en la consistencia con las bases de datos y los reportes gubernamentales que precisamente movilizan de forma explícita la versión estatal sobre el conflicto armado. De esta forma, el FUD legitima una versión del conflicto y una noción de víctima promovida por uno de sus actores: el propio Estado colombiano.

Dentro del mismo ejercicio reflexivo inspirado en Stengers, las relaciones de poder que se configuran a través del FUD nos conectan con distintas invitaciones, algunas de las cuales he seguido hasta el momento como la sugerida por Latour (2004) que redimensiona los objetos técnicos como asuntos de interés y señala su carácter mediador que le permite al FUD "traducir, redefinir, desplegar e incluso traicionar" aquello que ensambla (Latour, 1993, p. 81). Por su parte, Donna Haraway (1991) nos invita a denunciar las visiones de mundo que se promueven como objetivas, neutras, rigurosas y quizás libres de influencia social, cultural y política. En este caso la noción de víctima podría entenderse como una de esas visiones de mundo, que a partir de su legitimación experta en distintas cadenas de traducción, se logra naturalizar como un procedimiento dificilmente cuestionable. Este aspecto resulta más álgido y adquiere matices éticos y políticos importantes cuando las historias de personas afectadas por crimenes del Estado colombiano o de familiares de personas desmovilizadas carecen de pruebas según el FUD y los funcionarios. Aunque existen rutas alternas al sistema de reconocimiento de víctimas para el procesamiento legal de tales crimenes, me cuestiono la asimetría de tal ensamblado.

Sobre el anterior aspecto, Maria Puig de la Bellacasa (2011) nos interpela a pensar sobre las personas afectadas por relaciones de violencia que exceden la noción de víctima como actores que "pueden ser dañados con un ensamblado pero cuyas voces son menos valoradas, así como sus intereses y necesidad de cuidado" (p 92). En este sentido, 
la redistribución del bien de la confianza (Ashmore y Restrepo-Forero, 2013) por parte del sistema experto resulta perjudicial para algunas de las personas afectadas que deciden mantenerse fuera de los limites del reconocimiento estatal. Asimismo el acto de suministrar pruebas desdibuja la magnitud de las historias de violencia de los declarantes sometiéndolas a escrutinio técnico y reduciéndolas a códigos o anotaciones que ni siquiera constituyen aspectos de relevancia en la decisión sobre el RUV. Igualmente, el tiempo de circulación del FUD dentro de los nodos de evaluación genera un efecto nocivo que se suma a las dificiles experiencias diarias de muchos declarantes quienes en su mayoría acaban de migrar hacia las ciudades.

\section{Redefiniendo el reconocimiento de victimas}

Este trabajo ha permitido entender algunas de las herramientas técnicas a través de las cuales el Estado obtiene y organiza la información de personas que desean ser reconocidas como víctimas. Sería posible pensar en tal noción como un nuevo estatus que tiene lugar solo a partir del ensamblado entre expertos, formatos, declarantes y Estado. Pero también podríamos pensar que el reconocimiento, además de ser un acto de gestión administrativa, constituye un espacio de configuración socio-técnica de la noción estatal de víctima.

En las traducciones que conforman los procedimientos administrativos, los funcionarios, la UARIV y el Estado colombiano se dotan del poder de hablar en nombre de los registrados con el fin de dirigir la voz de las víctimas hacia otros sectores como la opinión pública, el Congreso, entidades privadas de derechos humanos, las actuales negociaciones de paz e incluso la comunidad internacional. Los caracteres comunes en los anteriores escenarios son la cifra oficial del RUV y los indicadores de gestión, reparación y presupuestos invertidos, entre otros, que se construyen desde el preciso instante en que los declarantes participan en las filas de los centros de asistencia. 
Además de cuestionar el estatus socio-técnico concedido a las víctimas como una condición para la restitución de sus derechos, una aproximación a las prácticas que construyen el RUV permite reformular el reconocimiento como una dinámica de agenciamiento mutuo entre las personas representadas dentro del RUV, los funcionarios públicos o expertos, los procedimientos burocráticos, los sistemas de información y las distintas instancias gubernamentales implicadas.

En el caso específico del FUD, tal dispositivo se intersecta con otros como el Plan de Asistencia, Atención y Reparación Individual, los distintos protocolos de atención psicosocial implementados también por agentes estatales y los planes de capacitación en proyectos productivos acompañados por funcionarios públicos. Un rastreo posterior de tales dispositivos, sus continuidades y discontinuidades podría sugerir elementos que permitan determinar las condiciones de Estado que tales dispositivos intervienen/construyen. Esto último representa una posibilidad para los Estudios de la Ciencia y la Tecnología y los estudios del Estado al dirigir su atención a los efectos performativos de la experticia y los dispositivos técnicos que participan en la configuración de formas de Estado en distintas localidades.

El estudio de tecnologías de representación como el FUD permite una aproximación a las condiciones estatales que se configuran precisamente a partir de tales artefactos. Igualmente, el estudio de las prácticas de configuración socio técnica del Estado permite redimensionar los cuestionamientos sobre la manera como el poder se institucionaliza y circula en distintos escenarios, pero también para señalar la múltiple direccionalidad de las relaciones entre distintos actores, incluyendo al Estado mismo como una entidad relacional.

Precisamente desde una perspectiva relacional sobre la configuración del Estado y una comprensión del reconocimiento como entidad socio técnica, podríamos sugerir que los actores que ofrecen sus narrativas para que sean representadas y traducidas dentro del FUD también están reconociendo y dotando al Estado de un nuevo estatus, en tanto dicho ensamblado promueve una versión exitosa de la gestión estatal de derechos humanos ante la opinión pública y la comunidad 
internacional. Tal promoción del Estado ocurre de manera independiente del resultado de la evaluación del FUD, puesto que los casos registrados y descartados se incorporan dentro de indicadores de gestión obtenidos a través de procedimientos 'objetivos y rigurosos'. El anterior planteamiento sobre la reconfiguración del Estado que resulta de la implementación del RUV puede ser enmarcado dentro de las apreciaciones de Mitchell (1991, p. 93 citado por Passoth y Rowland, 2010) que considera al Estado como el efecto de mecanismos de organización e intervención en las poblaciones más que como su fuente.

Con el fin de explorar posibles rutas futuras de investigación, he sugerido que los ensamblados estatales y de indicadores de gestión que circulan públicamente también invisibilizan, desplazan y traducen distintas experiencias y actores. En el anterior ensamblado, tecnologías como el RUV y el FUD constituyen un sector del complejo conjunto de relaciones, prácticas y procesos interrelacionados que reconfiguran el Estado colombiano como una entidad emergente. Así, desde las formulaciones de Passoth y Rowland (2010) sobre el estudio del Estado desde una perspectiva actor-red, propongo que tal abordaje relacional ofrece una aproximación alternativa a las instituciones y estructuras políticas que reta una concepción del Estado como un agente estático y ostensible. En tales abordajes es preciso enfatizar el carácter performado e inestable de los procesos socio técnicos de gobernabilidad de los cuales el reconocimiento de víctimas en Colombia es un ejemplo plausible.

Dentro de dicha comprensión alternativa del Estado, Ian Passoth y Nicholas Rowland (2010) han propuesto que el Estado "no es una cosa sino el resultado relacional en curso de prácticas y procesos que iterativamente lo expanden y protegen mientras otros lo disuelven y desentrañan" (p. 829). Así un Estado actante solo existe "en relación con ensamblados entre cuerpos humanos, fronteras y cercas, pago de impuestos y formas gubernamentales", así la propuesta de Passoth y Rowland (2010) considera al Estado como la organización que surge dentro de las prácticas. 
Resulta interesante pensar el reconocimiento de víctimas del conflicto precisamente como una condición de Estado que ensambla una racionalidad y objetividad alrededor de la cual se produce una nueva forma de organización estatal. Tal organización ha permitido que el Estado colombiano sea reconocido en distintos ámbitos como un Estado que "busca la paz" (The Daily Mail, 2014), "ha saldado una deuda histórica" (Semana, 2014), y que ha pasado "de capital del asesinato a central tecnoeléctrica". Dentro del anterior reconocimiento, existen otras tensiones que constituyen posibles rutas de interés investigativo como la participación política de víctimas. Por ejemplo en el mes de junio del 2014 la Mesa Nacional de Víctimas (MNV) conformada por 52 personas afectadas por distintos grupos armados e incluso por el Ejército Nacional, seleccionó en compañía de la dirección de la UARIV las primeras quince personas que viajarian a la mesa de negociación con las FARC-EP en la Habana. Dentro de los seleccionados habian diez personas reconocidas como víctimas escogidas a partir de la taxonomía de hechos victimizantes consignados en el FUD. A partir de la gestión de la MNV, se asignaron los cinco cupos restantes para personas afectadas por acciones del Ejército. Ante dicha selección, un grupo de 270 personas, solicitó reunirse públicamente con la MNV y la dirección de la UARIV en la cual manifestaron:

Somos las víctimas directas del grupo con el que se está negociando. Nosotros sentimos que deberíamos estar en esa discusión y que, en todos los espacios de participación, el Gobierno nos ha venido invisibilizando. Al diálogo con las FARC no se le deben mezclar otros componentes ni víctimas que no les corresponden [...] no nos sentimos representados porque no están las verdaderas víctimas de todos los crímenes de las FARC [...]. (Bermúdez-Liévano, 2014)

Así, se tuvieron que crear más delegaciones que no solo incluyeran personas reconocidas como víctimas de los hechos victimizantes contenidos en el FUD y personas afectadas por acciones del Ejército, sino también a líderes de movimientos organizados y líderes políticos que reclamaron por su situación. El cuestionamiento sobre la representatividad del FUD y su resultado en el RUV en el ámbito público y de negociaciones politicas resulta de interés en tanto denota el carácter 
no terminado e inestable del FUD como dispositivo que aún carece de reconocimiento como herramienta objetiva. Sin embargo, vale la pena destacar que el reclamo de falta de representatividad se dirigió al procedimiento de selección y no al carácter técnico del FUD o del RUV, de esta forma las trayectorias rastreadas hasta el momento constituyen insumos que nos permiten redimensionar el reconocimiento de víctimas del conflicto armado como un ensamblado socio-técnico, e indagar por otras dinámicas que se intersectan con este proyecto estatal.

\section{Referencias}

Ashmore, M. y Restrepo-Forero, O. (2013). El documento en su paso por la notaría: confianza, formalidad y credibilidad en Colombia. En O. Restrepo-Forero, (Ed.) Proyecto Ensamblado en Colombia. Tomo 2. Ensamblando heteroglosias (pp. 406-428). Bogotá, Colombia: Universidad Nacional de Colombia, Facultad de Ciencias Humanas, Centro de Estudios Sociales (CES), Grupo de Estudios Sociales de la Ciencia, la Tecnología y la Medicina(GESCTM).

Bermúdez-Liévano, A. (2014, julio 9). El pulso entre las víctimas por ir a La Habana. La Silla Vacía. Recuperado de http://lasillavacia.com/historia/elpulso-entre-las-victimas-por-ir-la-habana-48056.

Biehl, J., Good, B. y Kleinman, A. (2007). Subjectivity: ethnographic investigations. California: University of California Press.

Boyden, J. y De Berry, J. (2004). Children and youth on the front line: Ethnography, armed conflict and displacement. New York: Berghahn Books.

Brown, S. D. (2012). Two minutes of silence: Social technologies of public commemoration. Theory \& Psychology, 22(2), 234-252.

Callon, M. (1986). Some Elements of a Sociology of Translation: Domestication of the Scallops and the Fishermen. Power, Action and Belief: A New Sociology of Knowledge. London: Routledge \& Kegan. 
Callon, M. (2006). What does it mean to say that economics is performative? En D. MacKenzie, F. Muniesa y L. Siu, L. (Eds.). Do economists make markets? (pp. 311-357). Princeton, NJ: Princeton University Press.

Callon, M. y Latour, B. (1981). Unscrewing the big Leviathan: how actors macrostructure reality and how sociologists help them to do so. En Knorr-Cetina y Cicourel, A. (Eds.). Advances in Social Theory and Methodology: Toward an Integration of Microand Macro-Sociologies (pp. 277-303). London: Routledge y Kegan Paul.

Congreso de la República (2011, junio 10). Ley 1448. Ley de Víctimas y Restitución de Tierras. Diario oficial. Bogotá: Congreso de la República.

Cruz Roja Colombiana (2012). Formato Único de Declaración para la solicitud de inscripción en el Registro Único de Víctimas, Instrucciones para el diligenciamiento. Bogotá, Colombia: Cruz Roja.

DPS (2012). Cartilla de Diligenciamiento FUD Paso a Paso Version 03. Bogotá: DPS,

Foucault, M. (1983). Vigilar y castigar: nacimiento de la prisión. Buenos Aires: Siglo XXI.

Haraway, D. (1991). Simians, cyborgs, and women: The reinvention of women. London and New York: Routledge.

Harrell-Bond, B. E. (1986). Imposing aid: emergency assistance to refugees. Oxford: University Press Oxford.

Hess, D. (2001). Ethnography and the development of science and technology studies. En Harper y Lawson (Eds.) Handbook of ethnography (pp. 234-245). London: Sage.

Honneth, A. (2003). Redistribution as recognition: a response to Nancy Fraser. En Fraser y Honneth (Eds.) Redistribution or recognition (pp. 110-197). London: Verso.

Hull, M. S. (2012). Government of paper: The materiality of bureaucracy in urban Pakistan. California: University of California Press. 
Latour, B. (1993). The pasteurization of France. Harvard: Harvard University Press.

Latour, B. (1994). On technical mediation. Common knowledge, 3(2), 29-64.

Latour, B. (2004). Why has critique run out of steam? From matters of fact to matters of concern. Critical Inquiry, 30(2), 225-248.

Latour, B. (2005) Reassembling the social-an introduction to actor-network-theory, Subjectivity, Oxford: University Press.

Marcelo, G. (2013). Recognition and Critical Theory today. An interview with Axel Honneth. Philosophy \& Social Criticism, 39(2), 209-221.

Mora-Gámez, F. A. (2013). Reconocer a los reclamantes: sobre el Registro Único de Víctimas en Colombia como ensamblado socio técnico. IM-Pertinente, 1(1), 11-32.

Muniesa, F., Millo, Y. y Callon, M. (2007). An introduction to market devices. The sociological review, 55(s2), 1-12.

Papadopoulos, D., Stephenson, N. y Tsianos, V. (2008). Escape routes: control and subversion in the twenty-first century. London: Pluto Press.

Papadopoulos, D. y Tsianos, V. S. (2013). After citizenship: autonomy of migration, organisational ontology and mobile commons. Citizenship studies, 17(2), 178-196.

Passoth, J. H. y Rowland, N. J. (2010). Actor-network state integrating actornetwork theory and state theory. International Sociology, 25(6), 818-841.

Petryna, A. (2002). Life exposed: biological citizens after Chernobyl. Princeton: Princeton University Press.

Puig de la Bellacasa, M. (2011). Matters of care in technoscience: Assembling neglected things. Social Studies of Science, 41(1), 85-106. 
Rancière, J. (1998). Disagreement: Politics and philosophy. Minnesota: University of Minnesota Press.

Semana (2014, noviembre 15). Víctimas, según Harvard. Revista Semana. Recuperado de http://www.semana.com/nacion/articulo/victimas-segunharvard/409227-3

RNI (2015). Reporte general de la Red Nacional de Información. Colombia: Red Nacional de Información.

Schavelzon, S. (2007). El Gestionismo y el Politicismo en el Estado: el caso de dos estilos de gobierno en la Comisión Municipal de la Vivienda de la ciudad de Buenos Aires. Campos, Revista de Antropología Social, 8(2), pp. 41-63.

Schavelzon, S. (2010). La antropología del estado, su lugar y algunas problemáticas. Publicar, 8(9), 73-96.

Scott, J. C. (1998). Seeing like a state: How certain schemes to improve the human condition have failed. Connecticut: Yale University Press.

Stengers, I. (2002). Penser avec Whitehead: une libre et sauvage création de concepts Paris: Seuil.

The Daily Mail (2014, agosto 7). Colombia's president seeks peace deal in 2nd term. The Daily Mail. Recuperado de http://www.dailymail.co.uk/wires/ap/ article-2719256/Colombias-president-seeks-peace-deal-2nd-term.html

Tiscornia, S. (2004). Burocracias y violencia, Estudios de antropología politica. Buenos Aires: Antropofagia.

UARIV (2013). Personería de Bogotá y Unidad para las Víctimas en 'twitcam". Bogotá: UARIV.

UARIV (2015). Registro Único de Víctimas. Bogotá: UARIV.

Vikkelsø, S. (2012). The fragility of a robust therapeutic technique: The case of Bion's experience group. Theory \& Psychology, 22(2), 213-233. 
Cómo citar este artículo

Mora-Gámez, F. (2016). Reconocimiento de víctimas del conflicto armado en Colombia: Sobre tecnologías de representación y configuraciones de Estado. Universitas Humanística, 82, 75-101. http://dx.doi.org/10.11144/Javeriana. UH82.rvca 